\title{
Lessons Learned on IDEA from the Astro2020 Decadal Survey
}

This white paper is submitted as part of a collaborative effort organized by the Equity, Diversity, and Inclusion Working Group (EDIWG), a cross Assessment Group (AG) committee.

\section{Corresponding Author: Christina R. Richey, Jet Propulsion Laboratory, California Institute of Technology, (818)354-4940, christina.r.richey@jpl.nasa.gov}

\section{Co-Authors:}

Matthew S. Tiscareno, SETI Institute, matt@seti.org

Parvathy Prem, Johns Hopkins Applied Physics Laboratory, parvathy.prem@jhuapl.edu

Aparna Venkatesan, University of San Francisco, avenkatesan@usfca.edu

Julie Rathbun, The Planetary Science Institute, rathbun@psi.edu

Maggie McAdam, NASA Ames Research center, maggie.mcadam@nasa.gov

The Endorsement List contained $40+$ names at the time of submission. The Endorsement form will stay open after submission.

Endorsements as of submission: Edgard G. Rivera-Valentín (LPI/USRA), Rachel B. Fernandes (LPL, UArizona), Sarah E. Moran (JHU), Marshall J. Styczinski (UWashington), Jennifer L. Piatek (CCSU), Jennifer (JA) Grier (PSI), Maitrayee Bose (ASU), Barbara A. Cohen (NASA GSFC), James H. Roberts (JHU APL), Timothy A. Goudge (UT Austin), Timothy A. Livengood (UMCP), Michael A. Mischna (JPL/Caltech), Jennifer Hanley (Lowell Observatory), Michael C. Nolan (UArizona), Cynthia Phillips (JPL/Caltech), Michael W. Busch (SETI), JC Holbrook (University of the Western Cape), Veronica J. Bray (UArizona), Krista Soderlund (UT Austin), Devanshu Jha (MVJ College of Engineering,India), Martha Gilmore (Wesleyan University), Jonathan Lunine (Cornell University), Cecilia Leung (JPL/Caltech), Tony Freeman (JPL/Caltech), T. A. Nordheim (JPL/Caltech), Ariel N. Deutsch (Brown University), Sabine Stanley (JHU), Melissa G. Trainer (NASA GSFC), Nicole Whelley (NASA GSFC, UMCP), Jennifer Scully (JPL/Caltech), Alessondra Springmann (UArizona), Antonio J. Ricco (NASA ARC \& Stanford), Linda Spilker (JPL/Caltech), Jamie L. Molaro (PSI), Marisa Mayer (Stanford), Jeffrey M. Moore (NASA ARC), Alfred S. McEwen (UArizona), Floor Broekgaarden (Harvard \& Smithsonian CfA), Terry A. Hurford (NASA GSFC), Kunio M. Sayanagi (Hampton University), Margaret E. Landis (UCB/LASP), Noam R. Izenberg (JHU APL, VEXAG Deputy Chair), Abbie Grace (University of Tasmania), Zahra Khan (Independent Researcher), Tim Lister (Las Cumbres Observatory)

Acknowledgements: A portion of the work described was carried out at the Jet Propulsion Laboratory, California Institute of Technology, under a contract with the National Aeronautics and Space Administration (80NM0018D0004). 
Introduction: Task \#9 of the ongoing Planetary Science and Astrobiology Decadal Survey 2023 (hereafter Planetary2023) is to provide a clear exposition of "[t]he state of the profession including issues of diversity, inclusion, equity, and accessibility, the creation of safe workspaces, and recommended policies and practices to improve the state of the profession." ${ }^{1}$ This is similar to the task undertaken by the Decadal Survey on Astronomy and Astrophysics 2020 $(\text { Astro2020) })^{2}$ to assess the state of the profession. Astro2020, which will provide recommendations for the next decade of astrophysics research and is currently in the drafting phase of the study, received 294 White Papers concerning Activities, Projects, and the State of the Profession (APC).

Here we summarize 15 of the Astro2020 white papers that pertain to issues of IDEA (Inclusion, Diversity, Equity, and Accessibility, also referred to as DEIA). We find that many of these are relevant and even critical to Planetary2023, as much as they were to Astro2020. The current impact of the Covid-19 pandemic on the planetary science process is well documented ${ }^{3}$. In contrast to Astro2020, Planetary2023 is occurring during a global pandemic and worldwide protests against systemic racism. This has revealed the vulnerabilities around race, gender, economic disparities and lack of accessibility, and the disproportionate fallout for those most at risk in our field anticipated by many of the Astro2020 IDEA white papers ${ }^{4}$.

Recommendation \#1: The Planetary2023 Decadal panel should carefully consider the recommendations of Astro2020 APC White Papers that addressed needed steps towards a more inclusive, accessible, fair and healthy astronomy profession, as summarized by this white paper.

Recommendation \#2: The Planetary2023 panel should include the expertise of social scientists and others who have a track record of working to promote IDEA within the Planetary Science and Astrobiology community and/or within the STEM community more generally.

All papers below are Astro2020 APC White Papers that address IDEA issues applicable to the planetary science community.

1.https://www.nationalacademies.org/our-work/planetary-science-and-astrobiology-decadal-survey-2023-2032 2https://www.nationalacademies.org/our-work/decadal-survey-on-astronomy-and-astrophysics-2020-astro2020 3.https://womeninplanetaryscience.wordpress.com/2020/05/14/what-are-the-impacts-of-performing-a-decadal-su rvey-during-a-global-pandemic

4http://womeninastronomy.blogspot.com/2020/07/the-fallout-from-covid-19-on-astronomys.html 
\#1: Findings and Recommendations from the AAS Committee on the Status of Women in Astronomy: Towards Eliminating Harassment in Astronomy ${ }^{5}$

Lead Corresponding Author: Nicolle Zellner, Albion College, nzellner@albion.edu

Summary: The Committee on the Status of Women in Astronomy (CSWA) is calling on federal science funding agencies to take actions that will end harassment, particularly sexual harassment, in astronomical workplaces.

Recommendations: 1 . Federal agencies should improve their ethics policies by making harassment a form of scientific misconduct; 2 . Federal agencies should mandate that institutions report to them when a funded Principal Investigator (PI) or co-Principal Investigator (co-PI) is found to be a perpetrator of harassment; 3 . Federal funding agencies should provide online guides to help scientists identify harassment and connect them to the right resources for making confidential or official reports; 4 . Federal agencies should create and ensure the implementation of anti-harassment trainings by making them a requirement of receiving grant funding.

\section{\#2: Providing a Timely Review of Input Demographics to Advisory Committees ${ }^{6}$}

Lead Corresponding Author: Dara Norman, NOAO, dnorman@noao.edu

Summary: This is a white paper focused on why demographics and diversity studies are important to the community.

Recommendations: Advisory committees that collect community input (e.g., the Decadal Survey review committee) should also collect, compile and review input demographic data before finalizing reports (e.g., the final 2020 Decadal Survey Report). A summary of these data should be released alongside the final survey report.

\section{\#3: Tying Research Funding to Progress on Inclusion ${ }^{7}$}

Lead Corresponding Author: Dara Norman, NOAO, dnorman@noao.edu

Summary: This white paper focuses on the importance of diversity when building teams. The paper includes examples of metrics on which to judge DEIA impact and comments on the NSF's "Broader Impacts" strategic plan.

Recommendations: The main recommendation is that agency funding (from NSF, NASA, DOE, etc.), especially for missions, projects and programs, encourage the development and retention of diverse teams by requiring documentation of and progress on metrics related to diversity, inclusion and equity.

5http://survevgizmoresponseuploads.s3.amazonaws.com/fileuploads/623127/5043187/118-024ae14025ad4e36ac 10eff43489a5c5 ZellnerNicolle1.pdf

6http://surveygizmoresponseuploads.s3.amazonaws.com/fileuploads/623127/5043187/176-a9e6b61bc5a30f985cf 38a46e8f6e5d3 NormanDaraJ.pdf

Zhttp://survevgizmoresponseuploads.s3.amazonaws.com/fileuploads/623127/5043187/184-26dad8d099e513e19e 7fd755ecc3ea14_NormanDara.pdf 


\section{\#4: Why Physicists Should Discuss Mental Health ${ }^{8}$}

\section{Lead Corresponding Author: Kathryn Chandra, UCSD, kachandr@ucsd.edu}

Summary: This is a powerful and courageous personal account from an undergraduate student of what it is like to live with mental illness. There is no explicit list of recommendations, but the points below summarize some of the ideas and suggestions in the paper.

Recommendations: 1 . There is a need to increase and normalize discourse about mental disorders: what they are, and how severely they impact those affected; 2 . Programs should proactively address imposter syndrome by teaching students basic skills that they can apply to create a more positive and healthier mindset when tackling obstacles; 3 . Graduate admissions should include components, such as interviews, that allow students with poor transcripts and standardized test scores an opportunity to demonstrate their skills; 4. Educate students about the possibility of burnout and the signs of decreasing mental health as a way to strengthen both self-awareness and collaborations.

\section{\#5: Enhancing Conference Participation to Bridge the Diversity Gap ${ }^{9}$ Lead Corresponding Author: Laura Pritchard, STScl, Iprichard@stsci.edu}

Summary: This paper presents and analyzes the results of a survey on conference participation conducted at the Space Telescope Science Institute.

Recommendations: 1 . Enable two-way remote participation at every conference; 2. Conferences should have a diverse Science Organizing Committee and speaker list to achieve greater diversity of attendees; 3 . Organizers should consider allocating flexible funding for any additional dependent care costs incurred as a result of participating in a conference; 4. Wherever possible, institutions and conferences should allocate additional, targeted funding to enable conference attendance; 5 . The community should be surveyed to capture experiences that are not addressed in this survey.

\section{\#6: The Nonbinary Fraction: Looking Towards the Future of Gender Equity in Astronomy ${ }^{10}$ Lead Author: Kaitlin C. Rasmussen, University of Notre Dame Corresponding Author: Beck Stauss, Beck.E.Strauss@gmail.com}

Summary: This paper addresses the future of gender equity in astronomy by recommending survey practices and institutional policies based on a more complex approach to gender. Note this paper was also modified and resubmitted to Planetary2023.

Recommendations: 1 . Acknowledge and reconsider methodological choices that view gender as observable and discrete, or dismiss qualitative data such as participant testimony as

8http://survevgizmoresponseuploads.s3.amazonaws.com/fileuploads/623127/5043187/66-296be671b781d54195 a56ecb4bb3a94f ChandraKathrynT.pdf

2http://surveygizmoresponseuploads.s3.amazonaws.com/fileuploads/623127/5043187/137-03f2236af308dedbd5 22d487be61e587 PrichardLauraJ.pdf

10 http://survevgizmoresponseuploads.s3.amazonaws.com/fileuploads/623127/5043187/201-34a817a88cf462f834 47c91f08ee7df1_RasmussenKaitlinC.pdf 
statistically insignificant; 2 . Avoid gathering gender data through any means other than voluntary self-identification; 3. Enable compensated involvement by trained social scientists when studying marginalized people in astronomy; 4. Explicit and rigorous precautions should be taken to protect participant privacy when collecting gender data; 5 . Disciplines such as gender studies, critical race theory, ethics, and STSS (Science, Technology and Society Studies) should be part of undergraduate and graduate curricula, and workforce development programs.

\section{\#7: Astro2020 must issue actionable recommendations regarding diversity, inclusion, and} harassment $^{11}$

Lead Corresponding Author: Jane Rigby, NASA Goddard Space Flight Center, jane.rigby@nasa.gov

Summary: This white paper highlights that Astro2020 must issue actionable recommendations about diversity, inclusion, and harassment. The paper also outlines the previous method for State of the Profession (findings) as failed.

Recommendations: Issue concrete, actionable recommendations to funding agencies on how to support diversity and inclusion within our profession, how to stop harassment of all kinds, and how to respond as a community to those who have committed acts of harassment or discrimination

\#8: Leadership and Participation in NASA's Astrophysics Explorer-Class Missions ${ }^{12}$ Lead Corresponding Author: Joan Centrella, NASA Goddard Space Flight Center, joan.centrella@nasa.gov

Summary: This paper analyzes the (binary) gender breakdown of PIs and science teams responding to solicitations for NASA's Astrophysics Explorer-class missions between 2008-16. The authors find that women are significantly underrepresented, and that a significant proportion of teams consisted solely of men.

Recommendations: While the paper does not include specific recommendations, the authors urge that the committee consider the question of why women are so significantly underrepresented, and the consequences for the field.

\#9: A Different Kind of Dark Energy: Evidence for Placing Race and Gender in Physics ${ }^{13}$ Lead Corresponding Author: Lauren Marietta Chambers, STScl, lauren.m.chambers96@gmail.com

Summary: This paper examines the impact of race and gender on ways of knowing in physics and astronomy, spotlighting historical correlations between diversity and innovation, and how

${ }^{11}$ http://surveygizmoresponseuploads.s3.amazonaws.com/fileuploads/623127/5043187/34-51a0bc3115c7f2ff550 03a38e19f0e2c RigbyJaneR v2.pdf

12 http://surveygizmoresponseuploads.s3.amazonaws.com/fileuploads/623127/5043187/137-b53376610434e1a84 203e15453f60dd3 CentrellaJoan.pdf

13 http://survevgizmoresponseuploads.s3.amazonaws.com/fileuploads/623127/5043187/161-caee023ab4d2dc487 0698db6db7e4786 ChambersLaurenM.pdf 
colonial and masculine mindsets manifest in physics and astronomy. The author holds dual degrees in Astrophysics and African American Studies from Yale University, and this paper is an adaptation of their prize-winning undergraduate thesis.

Recommendations: 1 . Teach science and technology studies alongside science and technology; 2. Build a physics professoriate that is fluent in social justice; 3 . Critically examine the origins and costs of scientific work; 4. Embrace interdisciplinarity; 5. Encourage acknowledgement of positionality and subjectivity, and expand what kinds of perspectives and ideologies are valued.

\section{\#10: Proposed projects to improve inclusivity and equity in Astronomy outside of Departments $^{14}$ Lead Corresponding Author: Anonymous}

Summary: This anonymous white paper suggests that resources outside of universities be provided to report issues experienced by graduate students related to harassment, discrimination, bullying and other issues in astronomy departments. This white paper complements reports, e.g. by the AAS Task Force on Diversity and Inclusion in Astronomy Graduate Education, that contain resources *within* departments to combat these issues. Recommendations: 1 . An unbiased external organization that students could contact for support, anonymous reporting, overview of internal and external reporting process, resources and possible additional action. 2. Connect students who experience discrimination, harassment and other issues

\#11: Pursuing diversity, equity, and inclusion in multimessenger astronomy collaborations over the coming decade ${ }^{15}$

Lead Corresponding Author: Ellen Bechtol, UW-Madison, ellen.bechtol@icecube.wisc.edu Summary: Astronomy research is increasingly conducted in large research collaborations which are in the position to play a strong role in DEl efforts across all career stages. The Multimessenger Diversity Network (MDN), which includes a number of large scientific collaborations, is extending a cooperative model of research to DEI initiatives to improve DEI outcomes over the next decade.

Recommendations: The three initial recommendations of the MDN aim at systemic, rather than individual, changes to support sustainable DEl efforts: 1. Provide opportunities to share knowledge and resources on DEI-related activities; 2 . Include DEI in collaboration-level activities, including external reviews; and, 3. Develop and implement ways to recognize and concretely reward the DEI work of collaboration members. The authors recommend regular assessment and evaluation of these DEI efforts.

14http://surveygizmoresponseuploads.s3.amazonaws.com/fileuploads/623127/5043187/209-e0ed86188af934f313 e89c520faec651 BA.pdf

15 http://survevgizmoresponseuploads.s3.amazonaws.com/fileuploads/623127/5043187/198-16a32e0656f6aad3f7 ed03de0494ec37 BechtolEllenE.pdf 
\#12: Astrobites as a Community-led Model for Education, Science Communication, and Accessibility in Astrophysics ${ }^{16}$

Lead Corresponding Author: Gourav Khullar, University of Chicago, gkhullar@uchicago.edu Summary: This white paper describes the remarkable impact of Astrobites (an AAS-supported daily astrophysical literature digest written by graduate student volunteers) in reducing barriers to entry into astronomy research, and in promoting inclusive professional development.

Recommendations: 1 . Use resources like Astrobites in the classroom; 2. Promote platforms like Astrobites to reduce gatekeeping and increase diversity; 3. Develop Bites-based journal clubs; 4. Sponsor research on the efficacy of Bites programs; 5 . Increase financial and structural support for Bites programs; 6 . Increase support for graduate-student education in scientific communication.

\#13: Accessible Astronomy: Policies, Practices, and Strategies to Increase Participation of Astronomers with Disabilities ${ }^{17}$

Lead Corresponding Author: Alicia Aarnio, University of North Carolina Greensboro, anaarnio@uncg.edu

Summary: Individuals with disabilities are underrepresented in the labor force and in higher education. This white paper discusses the major barriers to access within the educational and professional practice of astronomy, and presents best practices for accessibility and inclusivity. Recommendations: The paper presents a detailed set of recommendations for professional societies, institutions, and funding agencies to address barriers that include: 1 . The lack of demographic data relating to disability; 2 . Lack of institutional support for accessibility (particularly at the graduate student and faculty levels) and insufficient recognition for inclusion work as prestigious and important; 3) Academic culture and hiring practices; 4 . Inaccessible spaces and information (including publications and datasets).

\section{\#14: Collaboration with Integrity: Indigenous Knowledge in 21st Century Astronomy ${ }^{18}$ Lead Corresponding Author: Aparna Venkatesan, University of San Francisco, avenkatesan@usfca.edu}

Summary: These authors share highly successful current initiatives based on a model of "collaboration with integrity" between western and indigenous scholars, and six key recommendations for US funding agencies that are important first steps for nonindigenous institutions to fully partner with indigenous communities. Collectively, these recommendations honor the intergenerational, interdisciplinary scientific research underlying indigenous

16http://survevgizmoresponseuploads.s3.amazonaws.com/fileuploads/623127/5043187/209-e6e8120eea317cc25 8347d3aa3a3c8fc KhullarGourav.pdf

${ }_{17}$ http://surveygizmoresponseuploads.s3.amazonaws.com/fileuploads/623127/5043187/183-7aaa4e556dd8cc99cf 2b19b79f7a0ffe AarnioAliciaN.pdf

18 http://surveygizmoresponseuploads.s3.amazonaws.com/fileuploads/623127/5043187/176-be5ca522e55decd1c 9b6f995ab81b704 VenkatesanAparna.pdf 
knowledge.

Recommendations: 1 . Create collaboration pathways for telescopes and other research facilities on indigenous lands; 2 . Dedicate funding for successful models that coherently synergize indigenous and nonindigenous science; 3 . Dedicate funding of long-term, observation-based interdisciplinary research conducted by indigenous knowledge holders; 4 . Recognize oral traditions as peer-reviewed, peer-verified scientific results; 5 . Dedicate conference funding for indigenous speakers and leaders; 6 . Create a culturally supported path for full participation of indigenous youth in science careers.

\section{\#15: A Hua He Inoa: Hawaiian Culture-Based Celestial Naming ${ }^{19}$}

Lead Corresponding Author: Ka'iu Kimura ('Imiloa Astronomy Center of Hawai'i, University of Hawai'i, Hilo), kaiukimura@gmail.com

Summary: The A Hua He Inoa (AAHI; "calling forth a name") nomenclature project is a partnership between the 'Imiloa Astronomy Center and Hawai'i-based astronomical observatories. Its purpose is to "weave traditional indigenous practices into the official naming of astronomical discoveries made in Hawai'i." Through this project, the interstellar object 'Oumuamua and the supermassive black hole Pōwehi were named in a spirit of co-creation and collaboration between astronomers and the Hawaiian community. Native Hawaiian youth are an integral part of the project, which teaches them to appreciate both scientific and traditional Hawaiian knowledge.

Recommendations: Continued support for AHHI's participating institutions and communities, and consider opportunities to "replicate and scale this effort" for other indigenous and astronomy communities nationally.

19 https://ui.adsabs.harvard.edu/abs/2019BAAS...51g.135K/abstract 